\title{
Measuring M dwarf Winds with DAZ White Dwarfs ${ }^{1}$
}

\author{
John H. Debes ${ }^{2}$
}

\begin{abstract}
Hydrogen atmosphere white dwarfs with metal lines, so-called DAZs, show evidence for ongoing accretion of material onto their surfaces. Some DAZs are known to have unresolved $M$ dwarf companions, which could account for the observed accretion through a stellar wind. I combine observed Ca abundances of the DAZs with information on the orbital separation of their M dwarf companions to infer the mass loss rate of the $\mathrm{M}$ dwarfs. I find that for three of the six known DAZs with M dwarf companions, a stellar wind can plausibly explain the observed accretion on the white dwarfs assuming Bondi-Hoyle accretion of solar abundance stellar winds on the order of $10^{-14}-10^{-16} \mathrm{M}_{\odot} \mathrm{yr}^{-1}$. The rest of the sample have companions with orbits $\gtrsim 1 \mathrm{AU}$, and require companion mass loss rates of $>$ $10^{-11} \mathrm{M}_{\odot} \mathrm{yr}^{-1}$. I conclude that there must be an alternative explanation for accretion of material onto DAZs with widely separated companions. The inferred winds for two of the close binaries are orders of magnitude smaller than typically assumed for the angular momentum loss of red dwarf-white dwarf pairs due to magnetic braking from a stellar wind and may seriously affect predictions for the formation rate of $\mathrm{CVs}$ with low mass companions.
\end{abstract}

Subject headings: circumstellar matter — stars: winds, outflows — white dwarfs - stars: late-type

\section{Introduction}

Hydrogen atmosphere white dwarfs with spectral absorption lines due to metals are a potential puzzle for white dwarf evolution. Metals present in the upper atmossheres of white

\footnotetext{
${ }^{2}$ Department of Terrestrial Magnetism, Carnegie Institution of Washington, Washington, DC 20015, debes@dtm.ciw.edu

${ }^{1}$ Based on observations made with the NASA/ESA Hubble Space Telescope, obtained from the Data Archive at the Space Telescope Science Institute, which is operated by the Association of Universities for Research in Astronomy, Inc., under NASA contract NAS 5-26555. These observations are associated with program 10255
} 
dwarfs with $\mathrm{T}_{\text {eff }}<20.000 \mathrm{~K}$ generally diffuse out of the upper atmosphere on timescales ranging from days to $10^{6} \mathrm{yr}$ (Paquette et al. 1986). It was therefore expected that white dwarfs with cooling ages $\gg 10^{8}$ yr should show no detectable metal line absorption. However a number of both hydrogen (class DA) and helium (class DB) white dwarfs show the evidence of heavy metals in their atmospheres (classes DAZ and DZ). The initial explanation for metal contamination was the accretion of solar abundance ISM material(Dupuis et al. 1993b, 1992, 1993a). But the lack of correlation between white dwarfs and dense ISM clouds, a large number of DAZs residing at $<70 \mathrm{pc}$ within the Local Bubble, the high inferred accretion rates for DAZs, and the lack of hydrogen in DZ atmospheres makes ISM accretion problematic(Aannestad et al. 1993; Zuckerman \& Reid 1998; Zuckerman et al. 2003). Recent surveys of white dwarfs have turned up several new DAZs, and these have been used to put forth a separate ISM accretion argument for within the Local Bubble (Koester et al. 2005; Koester \& Wilken 2006). In this case, nearby white dwarfs accrete continuously from clouds of warm, partially ionized ISM

Other explanations exist, however. The direct accretion of heavy metals through cometary impacts with white dwarfs was invoked to explain the first DAZ, G74-7 (Alcock et al. 1986). This explanation can deliver a moderate amount of material to the white dwarf surface, but fails to explain high abundance DAZs with short settling times and dusty disks such as G29-38 and GD 362 unless a planetary system perturbs a steady supply of material close to the white dwarf (Zuckerman \& Becklin 1987; Koester et al. 1997; Holberg et al. 1997; Debes \& Sigurdsson 2002; Gianninas et al. 2004).

In a survey of DAs for metal lines, six of ten known unresolved DA+M dwarf systems were DAZs compared to $1 / 9$ for double degenerates and $0 / 7$ for resolved binaries(Zuckerman et al. 2003). This raises the possiblity that DAZs may be caused by hitherto unseen stellar or substellar companions that deposit material through a stellar wind onto the surfaces of the white dwarfs. The evidence for apparently single DAZs with unknown companions is thin. Only two known substellar companions to white dwarfs exist, in widely separated binaries (Zuckerman \& Becklin 1992; Farihi \& Christopher 2004). This is despite wide searches for unresolved stellar or substellar objects in close orbits (Probst \& Oconnell 1982; Zuckerman \& Becklin 1992; Dobbie et al. 2005; Farihi et al. 2005). Furthermore, concerted searches of DAZs themselves show that stellar companions are ruled out at all separations and substellar companions ruled out for all but the closest orbital separations (Debes et al. 2005a,b, 2006).

M Dwarf stellar wind mass loss rates are poorly understood and hard to study-upper limits can only be placed on the nearby M5.5 Proxima Centauri based on X-ray and Ly $\alpha$ studies with upper limits of $6 \times 10^{-14} M_{\odot} / \mathrm{yr}\left(3 \dot{M}_{\odot}\right)$ and $4 \times 10^{-15} M_{\odot} / \mathrm{yr}\left(0.2 \dot{M}_{\odot}\right)$ respectively (Wargelin \& Drake 2002; Wood et al. 2001). Radio observations with marginal detections 
and spectroscopic observations of coronal mass ejections from low mass stars have been used to propose very high mass loss rates from dwarf stars, which has a theoretical motivation (Mullan et al. 1992; Houdebine et al. 1990; Badalyan \& Livshits 1992). These results are controversial, however, and depend on the model assumptions made (Lim \& White 1996). The recent results for Proxima Centauri suggest that perhaps $M$ dwarf stars have winds comparable to or smaller than solar type stars, rather than the larger rates. The strength of young $\mathrm{M}$ dwarf winds can affect the lifetime of gaseous material in disks that are important for planet formation and limit the presence of dusty disks (Laughlin et al. 2004; Plavchan et al. 2005). In orbit around white dwarfs, $M$ dwarf winds are important for the origin of cataclysmic variables (Schreiber \& Gänsicke 2003).

Under the assumption that the cause of the metal lines in DAZs is due to $\mathrm{M}$ dwarf companions, the stellar wind rates for known DAZ $+\mathrm{M}$ dwarf systems can be calculated. These calculations require the known orbits of the $M$ dwarf companions. Three of the known DAZ+M systems have well known orbital periods and masses since they are detached transiting systems. The other three have recent HST Advanced Camera for Surveys (ACS) images that allow orbital information to be estimated.

In this paper I infer the stellar wind mass loss rates of the $\mathrm{M}$ dwarf companions to six DAZ+M dwarf binaries. In Section 2 I estimate the orbital semi-major axis for each binary. In Section 3 I use the semi-major axes and white dwarf diffusion coefficients to infer the mass accretion rate onto the white dwarfs and thus the mass loss rate of the companion $\mathrm{M}$ dwarfs. Finally, in Section 4 I discuss my results.

\section{Determining Orbital Separations}

\subsection{Close Binaries}

Three of the six DAZs with companions have previously measured orbital periods, primary, and companion masses. WD $0419-487$ is a $0.47 \mathrm{M}_{\odot}$ white dwarf with a 0.095 companion in a $7.3 \mathrm{hr}$ orbit. The spectroscopic survey of DAs that discovered WD 0419-487 noted that there was evidence of a hot spot/accretion disk in the observed spectrum (Zuckerman et al. 2003). I would expect that this object may show a higher accretion rate due to some amount of mass transfer in addition to that caused by a wind. WD $1026+002$ is a $0.68 \mathrm{M}_{\odot}$ white dwarf with a $0.23 \mathrm{M}_{\odot}$ companion in a $14.3 \mathrm{hr}$ orbit. Finally, WD $1213+528$ is a $0.63 \mathrm{M}_{\odot}$ white dwarf with a $0.36 \mathrm{M}_{\odot}$ companion in a $16 \mathrm{hr}$ orbit. Table 1 lists the orbital parameters important for my calculations. 


\subsection{Widely Separated Binaries}

The remaining three systems have had their radial velocities measured to an accuracy within a few km/s and show either slow RV trends or none at all (Schultz et al. 1996). These WDs are prime candidates for high spatial resolution imaging in order to determine what their approximate orbital separations are. Recently, a large snapshot survey (Program 10255, PI D. Hoard) with the Hubble Space Telescope's Advanced Camera for Surveys (ACS) was performed on a large sample of previously unresolved white dwarf+red dwarf pairs, including the three DAZ + M systems that have no orbital information (for first results, see Farihi et al. 2006). Of these three, WD 1049+103 and WD 1210+464 show resolved companions with the ACS data. The third DAZ, WD 0354+463 shows no obvious resolved companion (Farihi et al. 2006).

The STScI pipeline calibrated ACS data for these three WDs was retrieved and analyzed by measuring the centroid positions of the white dwarf and resolved companion. Figure 1 shows the two imaged targets with resolved companions. The data was taken with the High Resolution Camera (HRC) on ACS and so the plate scale for the final geometrically corrected images is $\sim 0^{\prime} 025 /$ pixel. The projected radius was calculated and this was used to estimate an orbital separation assuming a circular orbit.

Since no resolved companion was detected for WD 0354+463, an estimate for the upper limit to the $\mathrm{M}$ dwarf orbital separation must be done. One can estimate the sensitivity of an image to a resolved companion by implanting artificial companions into the data and measure when they are recovered. I constructed a reference HRC PSF from the image of WD $1210+464$. At separations $>70$ mas or $2 \mathrm{AU}$, an object with $\mathrm{m}_{F 814 W}=15.4$ could have been detected, corresponding to the approximate F814W magnitude expected for both WD $0354+463$ and the $\mathrm{M}$ dwarf assuming a mass of $0.1 \mathrm{M}_{\odot}$ (Baraffe et al. 1998). Figure 2 shows the limiting separation at which WD $0354+463$ would have been observably separated from its companion.

As a lower limit to the orbital separation, I use the timescale of radial velocity observations by Schultz et al. (1996), which is $\sim 0.7$ yr. Assuming a primary white dwarf mass of $0.6 \mathrm{M}_{\odot}$, I calculated a lower bound on the orbital separation based on no observed variations. If I assume that at least half a period would have been detected and neglect the mass of the companion, I obtain a lower bound on the orbital semi-major axis of $\sim 1$ AU. The projected separation would be $\sim 35$ mas, well below the spatial resolution of ACS. I adopt a value of $1.5 \pm 0.5 \mathrm{AU}$ for the orbital separation of the M dwarf companion.

Finally, one cannot estimate an orbital separation without knowledge of the white

dwarf's distance from the Sun. None of these three objects have parallax measurements 
so I utilize the determinations of the distance based on model fits to broadband photometry. WD $0354+463$ 's distance can be calculated by an estimate of its absolute magnitude, which gives a distance of $\sim 30$ pc (McCook \& Sion 1999). For WD 1049+103, and WD 1210+464, both objects are part of the PG survey, which has been modeled by Liebert et al. (2005). The model distances and the estimated semi-major axes are listed in Table 2.

\section{Inferring Mass Accretion and Stellar Wind Rates}

Inferring the mass accretion onto the white dwarf proceeds from a knowledge of the number abundance of some tracer element relative to hydrogen. In the case of DAZs the strongest observed lines in the visible tend to be the $\mathrm{Ca} \mathrm{H}$ and $\mathrm{K}$ lines. If one assumes a solar composition of material accreted, the accretion rate is then

$$
\dot{M}=\frac{q M_{w d}[C a / H]}{\Theta[C a / H]_{\odot}}
$$

where $q$ is the mass fraction at the base of the convection zone and $\Theta$ is the e-folding timescale for Ca to settle out of the atmosphere (Paquette et al. 1986). $[\mathrm{Ca} / \mathrm{H}]_{\odot}$ is the solar value of $[\mathrm{Ca} / \mathrm{H}]$ or $2.1875 \times 10^{-6}$. I use the observed number abundances for the DAZs calculated in Zuckerman et al. (2003). For WD 1210+464, there is no abundance calculated, but based on the upper limits based on non-detections of other white dwarfs in that survey, I estimate that $[\mathrm{Ca} / \mathrm{H}] \sim-7$. I discuss this further in Section 4. It should be noted that based on its $\mathrm{T}_{\text {eff }}$, WD 1210+464 may be hot enough to sustain a radiative wind which would create metal lines, thus making it irrelevent that it has a companion. For completeness, I still calculate the inferred mass loss rate of its companion.

For this paper, I use the values of $q$ determined by Althaus \& Benvenuto (1998) and the values of $\Theta$ by Paquette et al. (1986), even though these two results use different methods of calculating turbulence. This method has been shown to produce the accretion rate for the DAZ G29-38 reasonably well in light of independent estimations of the accretion rate (Graham et al. 1990; Debes \& Sigurdsson 2002). Additionally for WD 1049+103 and WD $1210+464$, their $\mathrm{T}_{\text {eff }}$ are higher than those calculated by either method, so I use the values from the highest effective temperatures calculated.

Inferring the mass loss rate of the companion requires a mechanism for accretion. For simplicity and without knowledge of the exact mechanism for creating a stellar wind, I assume the $\mathrm{M}$ dwarf companion expels a spherically symmetric flow at the escape speed from the surface of the M dwarf. I assume that the white dwarf accretes material through a 
Bondi-Hoyle accretion flow determined by

$$
\dot{M}=\frac{4 \pi G^{2} M_{W D}^{2} \rho(R)}{v_{r e l}^{3}}
$$

where $G$ is the gravitational constant, $\rho$ is the density of material surrounding the WD, and $v$ is the relative velocity to the WD at which the gas is passing, which $\mathrm{I}$ take to be $\sqrt{v_{\text {wind }}^{2}+v_{\text {orb }}^{2}}$ (Bondi \& Hoyle 1944). The escape speed from a low mass star is $\sim 600 \mathrm{~km} / \mathrm{s}$, assuming that $M / R \sim 1$. It is possible that the widely separated binaries have a higher mass than the close binaries. The mass-radius relation is roughly true for most low mass stars as can be seen in Table 1 (but see López-Morales \& Ribas 2005). However, WD 0419487's radius is roughly twice as large as would be expected. This could be related to the possiblitity of its red dwarf undergoing mass transfer with the white dwarf, or the fact that it is most likely tidally locked and is a fast rotator. In any case, the assumption of $600 \mathrm{~km} / \mathrm{s}$ for an escape speed for the widely separated binaries I'm studying is probably safe, since they should be similar to field red dwarfs.

Using the escape speed at the radius of the companion may not be strictly true. For example, one could model isothermal or polytropic winds from the $\mathrm{M}$ dwarfs assuming some heating mechanism. This is highly model dependent and requires information about the $\mathrm{M}$ dwarf companions that is not easily determined by the observations at hand. Presumably the main source of heating for the companions is comparable to that for the solar wind, where coronal heating provides the bulk of energy for the acceleration of the wind. Uncertainties in $v$ are discussed further in Section 4.

Additionally, Bondi-Hoyle accretion may not accurately describe these systems. Many astrophysical objects show departures from the simple Bondi-Hoyle picture, such as isolated neutron stars accreting from the ISM (Perna et al. 2003) and super massive black holes (Di Matteo et al. 2001). In these cases, there are departures from the simple plane parallel geometry of the Bondi-Hoyle case due to turbulence, and magnetic fields (Krumholz et al. 2005). These departures serve to suppress the Bondi Hoyle accretion rate, making accretion more inefficient. Wind accretion in binary systems as well can depart from the simple BondiHoyle case, particularly when $v_{\text {orb }} \sim v_{\text {wind }}$. In these cases, the accretion rates can be an order of magnitude lower (Theuns \& Jorissen 1993; Theuns et al. 1996). For all of these situations, the accretion supression results in higher inferred wind mass loss rates. The values I calculate correspond to strict lower limits if simple Bondi-Hoyle accretion is not present.

The final step is to link the mass loss rate of the red dwarf to the observed accretion rate onto the WD. The density depends directly on this mass loss. I assume that the mass loss rate is constant and calculate the density due to the continuity equation (Lamers \& 
Cassinelli 1999):

$$
\rho(R)=\frac{\dot{M}_{R D}}{4 \pi v R^{2}}
$$

where $\dot{M}_{R D}$ is the mass loss rate. The equation can be combined with Equations 1 and 2 and solved for $\dot{M}_{R D}$ in terms of known or inferred quantities:

$$
\dot{M}_{R D}=\frac{q[\mathrm{Ca} / \mathrm{H}]}{\Theta[\mathrm{Ca} / H]_{\odot}} \frac{R^{2} v^{4}}{G^{2} M_{W D}}
$$

I tabulate my results for the known close binaries and the resolved binaries in Table 3 . For comparison, the solar wind mass loss rate is $\sim 2 \times 10^{-14} \mathrm{M}_{\odot} / \mathrm{yr}$.

The uncertainties in Equation 4 can be roughly quantified. The close binaries have more accurate measures of $\dot{M}$, since $\mathrm{M}_{W D}, R$, and $[\mathrm{Ca} / \mathrm{H}]$ are all known to within a few percent. In their cases the formal statistical error based on these measurements comes to $<10 \%$. For the widely separated binaries, the errors in $\mathrm{M}_{W D}$ and $[\mathrm{Ca} / \mathrm{H}]$ are on the order of $\sim 10 \%$ as well. Assuming that the model parameters and $v$ are correct, $\dot{M}_{R D}$ is well constrained.

Since the results are model dependent, systematic errors most certainly dominate over the statistical errors estimated above. Uncertainty is present in the calculation of $\Theta$, which depends on $q$, the mass fraction of the convective envolope, the diffusion coefficients used, and of the convective envelope, which is highly sensitive to certain ranges of temperature and depends on the formalism of turbulence chosen (see Althaus \& Benvenuto 1998, for example). At $\mathrm{T}_{\text {eff }}>12000 \mathrm{~K}$ and $<8000 \mathrm{~K}$, the various flavors of turbulence tend to converge within factors of a few, while between these two ranges values for $q$ vary by up to four orders of magnitude. This uncertainty is helped somewhat by the fact that $\Theta \propto q^{0.7}$ (Paquette et al. 1986). Equation 4 shows that $\dot{M}_{R D} \propto q^{0.3}$. Thus an uncertainty of up to four orders of magnitude in $q$ corresponds to a uncertainty in $\dot{M}$ of a factor of 15 . To self-consistently calculate $q$ and $\Theta$, the same turbulence formalism should be employed to determine both quantities.

Unfortunately, a self-consistent calculation of the diffusion times using different turbulences has yet to be synthesized properly for the range of temperatures, masses, and atmospheric compositions relevant to cool DAZs, and understanding of the underlying mechanism for forming DAZs would be greatly advanced by an update of this important work (see also Zuckerman et al. 2003). Most of the DAZs in my sample are in the range where the various models converge well to within factors of two, so the uncertainty in $q / \Theta \sim 25 \%$.

The distance $R$, determined from the estimated orbital separations can be uncertain for the widely separated binaries. Since these are projected distances, they do not necessarily correspond to the true orbital separation of the pair. However, any such uncertainty would 
serve to raise the inferred stellar wind mass loss rate since the projected orbital separation would always be smaller than the true orbital separation. Conversely, the companions could

be in high eccentricity orbits which brought the star much closer than observed. Such a situation would mean that the mass loss rate would be overestimated. In that case, $R$ would have to change by a factor of three (corresponding to an orbital $e \sim 0.5$ ) to roughly produce an order of magnitude change in $\dot{M}_{R D}$. It is not guaranteed that the resolved companions are physically bound since there is no common proper motion information-the observations were taken at one epoch. The extremely close separations $\left(<1^{\prime \prime}\right)$ and the fact that the blended near-IR photometry are consistent with companions at the same distance makes chance alignments unlikely.

The solar wind velocity is variable and ranges from $60 \%$ to greater than the escape speed of the Sun, implying $v$ could have a range of values that probably does not exceed a factor of two. The true velocity of the wind from an M dwarf depends sensitively on the model for that wind, which is beyond the scope of this paper. In the case of the solar wind, the measured velocity at $1 \mathrm{AU}$ is less than that predicted for an isothermal or polytropic wind (Lamers \& Cassinelli 1999). Since the inferred mass loss rate is $\propto v^{4}$, a change in estimated speed by a factor of two would change the calculated mass loss rates by an order of magnitude.

To sum up, systematic errors in $\dot{M}_{R D} \propto q^{0.3} R^{2} v^{4}$, with $v$ creating the largest systematic uncertainty for the close binaries and $R$ and $v$ creating the largest uncertainties for the resolved systems. The estimates presented in Table 3 are accurate to an order of magnitude for the close binaries while the resolved binaries are accurate to within two orders of magnitude. This is comparable to the range of upper limits given for the mass loss rate of Proxima Centauri, which approximately spans an order of magnitude.

\section{Discussion}

Our results break down along the lines of those DAZs with known close companions $(a<0.02 \mathrm{AU})$ and those with greater separations. At smaller separations, the calculated mass loss rates agree reasonably well with the upper limit to the wind around Proxima Centauri (Wargelin \& Drake 2002; Wood et al. 2002). The mass loss rates I calculate are about two orders of magnitude smaller than Proxima Centauri's smaller upper limit, with the exception of WD 0419-487 which is comparable, though larger than the other two M dwarfs. This larger rate could be explained by moderate Roche lobe overflow, evaporation of the companion by the DAZ, or efficient capture of the companion's wind by a magnetic field. If WD 0419-487 was efficiently capturing all of its companion's wind, $\dot{M}_{R D}$ would fall 
nicely in with those observed for the other two close binaries.

On the other hand, the mass loss rates determined for the widely separated companions are three to four orders of magnitude larger than the Solar wind. This is despite a slightly higher uncertainty of the accretion rate onto the white dwarfs. Most of these uncertainties would conspire to create a higher accretion rate. For WD 1210+464, a lower accretion rate is possible if the detected equivalent width corresponds to a lower abundance than assumed. However, even at the smallest lower limit of the Zuckerman et al. (2003) survey ([Ca/H] 12.8), the inferred mass loss rate of the companion would be equivalent to the Solar Wind and two orders of magnitude higher than the close binaries. It is possible that these systems are hierarchical triples with companions undetected by radial velocity observations but in orbits similar to the close binaries. WD $1210+464$ and WD1049+103 have F814W photometry consistent with single DAZs, neglecting their resolved companions. This strongly argues that any further unresolved companions would have to be quite dim and of low mass. Conversely, $\mathrm{M}$ dwarfs could have the super solar rates predicted by the earlier results, but in light of the estimated winds of Proxima Centauri and the three close $\mathrm{M}$ dwarfs this seems unlikely. Furthermore, given the inferred total ages of the host white dwarfs, the companions would have either completely evaporated or lost a large fraction of their total mass.

The low mass loss rates for the three closest binaries has two possible interpretations. Either the mechanism for accretion is suppressed relative to Bondi-Hoyle accretion by several orders of magnitude if one expects $M$ dwarf winds to be similar to the Sun, or M dwarf winds are quenched even in situations where they are rotating quickly and should have significant activity due to strong magnetic fields. Some evidence for the quenching of winds for low mass stars comes from Mohanty \& Basri (2003), who find that very late spectral type stars have lower indicators of activity due to a corona or chromosphere. This has been noted in studies of the angular momentum evolution of $\mathrm{CVs}$, where fully convective companions were believed to have lost less angular momentum due to an inefficient dynamo process (Durney et al. 1993).

If the winds from these low mass companions are orders of magnitdue smaller than the solar wind mass loss rate, this has significant implications for the angular momentum evolution of CV progenitors and CVs themselves. Typically, it has been assumed that the angular momentum evolution of a CV or CV-progenitor occurred due to magnetic braking through a stellar wind, with angular momentum loss:

$$
\dot{J}=-K_{w}\left(\frac{R_{\text {sec }}}{\mathrm{R}_{\odot}}\right)^{2-N}\left(\frac{M_{\text {sec }}}{\mathrm{M}_{\odot}}\right)^{-N / 3}\left(\frac{\dot{M}}{10^{-14} \mathrm{M}_{\odot} s^{-1}}\right)^{1-2 N / 3} \omega^{3} \text { for } \omega \leq \omega_{\text {crit }}
$$




$$
=-K_{w}\left(\frac{R_{\text {sec }}}{\mathrm{R}_{\odot}}\right)^{2-N}\left(\frac{M_{\text {sec }}}{\mathrm{M}_{\odot}}\right)^{-N / 3}\left(\frac{\dot{M}}{10^{-14} \mathrm{M}_{\odot} s^{-1}}\right)^{1-2 N / 3} \omega \omega_{\text {crit }}^{2} \text { for } \omega>\omega_{\text {crit }}
$$

where $\omega_{\text {crit }}$ represents a cutoff rotation speed where the magnetic field saturates. This equation is a modified form of that used by Kawaler (1988), following the work of Mestel \& Spruit (1987) and Weber \& Davis (1967). This prescription was used by others to estimate the timescale for the evolution of single low mass stars, the onset of mass transfer in CV progenitors, and the timescale for the evolution of CVs (Sills et al. 2000; Schreiber \& Gänsicke 2003; Andronov et al. 2003). In all of these cases, it was implicitly assumed that the mass loss rate was approximately the solar wind mass loss rate. $K_{w}$ and $N$ were essentially free parameters but were chosen to be $2.7 \times 10^{47} \mathrm{~g} \mathrm{~cm} \mathrm{~s}$ and 1.5 respectively in these works. $K_{w}$ essentially collects all the uncertainties in the properties of the wind and magnetic field of a particular star and $N$ corresponds to the particular magnetic field geometry. It is immediately apparent that for the given $N, \dot{J} \propto \dot{M}$ and the consequent angular momentum loss rate for the observed DAZs would be 1-2 orders of magnitude smaller than would be calculated by Equation 5. The consequent timescale for these binaries to become CVs would be dominated by gravitational radiation, and would not be affected significantly by magnetic braking.

I also investigate a plausible orbital separation cutoff where a typical measured WD accretion rate can be maintained by an $\mathrm{M}$ dwarf wind. Using a similar calculation as I used for my widely separated binaries, I place a cutoff for reasonable mass loss rates due to winds at the stellar wind upper limits for Proxima Centauri (See Table 3). Figure 3 shows the result for varying levels of mass accretion that should be typical for DAZs. The lowest accretion rates allow plausible companions at wider separations, but the maximum allowed is $\sim 1 \mathrm{AU}$.

It may be instructive to separate DAZs into three classes for ease of identifying the underlying mechanism for accretion. The first class would be DAZs with companions in orbits $\lesssim 1$ AU. These are most plausibly explained by a wind scenario and can be used to measure $\mathrm{M}$ dwarf winds to rates orders of magnitude smaller than other methods (Wood et al. 2001, 2002). The second class would encompass all singular WDs and WDs in binaries $\gtrsim 1$ AU, with distances from the Sun $<70$ pc. These DAZs cannot easily be explained by either ISM accretion or the companion wind explanation. The third class of DAZs would be at distances $\gtrsim 70 \mathrm{pc}$ and be either singular or have companions at separations $\gtrsim 1 \mathrm{AU}$. These objects would either have to show ISM accretion through correlation with a known dense cloud or through spectroscopic evidence of a surrounding medium. Otherwise they would have the same origin as those in the second class. 
ISM accretion remains viable for the two binaries that are at larger distances, though one would expect these two objects to correspond with known clouds or show some other evidence of a fairly dense ISM component. WD 0354+463, however, is well within the Local Bubble. Both wind accretion and ISM accretion would have difficulty explaining this object. Planetesimal accretion could still be possible. This can either occur through comet impacts and disruptions such as suggested by Alcock et al. (1986) and Debes \& Sigurdsson (2002), or through the tidal disruption of asteroids as suggested by Jura (2003). Relic planetesimals from a circumbinary ring around the two stars could provide the necessary materials. The M dwarf could be slowly perturbing the planetesimals. Since extrasolar planets are known to

exist in binary systems, the interaction between a planet and $\mathrm{M}$ dwarf could be perturbing planetesimals into WD crossing orbits. If this is the case, the resevoir of planetesimals might be detectable with Spitzer or at longer wavelengths.

Finally, there are roughly 15 other detached systems with M dwarf companions that have orbital information and masses for both the white dwarf and the companion that could be analyzed in the same way (Ritter \& Kolb 2003). Medium to high resolution optical spectra could be used to find out how many of these objects possess metal lines, and whether the mass loss rates inferred for those systems match the binaries in my sample.

This work has made extensive use of both the Simbad and Vizier services. This paper has been greatly improved by an anonymous referee. JD would like to thank Steinn Sigurdsson and Alycia Weinberger for extremely helpful comments and insights.

\section{REFERENCES}

Aannestad, P. A., Kenyon, S. J., Hammond, G. L., \& Sion, E. M. 1993, AJ, 105, 1033

Alcock, C., Fristrom, C. C., \& Siegelman, R. 1986, ApJ, 302, 462

Althaus, L. G. \& Benvenuto, O. G. 1998, MNRAS, 296, 206

Andronov, N., Pinsonneault, M., \& Sills, A. 2003, ApJ, 582, 358

Badalyan, O. G. \& Livshits, M. A. 1992, Soviet Astronomy, 36, 70

Baraffe, I., Chabrier, G., Allard, F., \& Hauschildt, P. H. 1998, Astron. Astrophys., 337, 403

Bondi, H. \& Hoyle, F. 1944, MNRAS, 104, 273

Bruch, A. 1999, AJ, 117, 3031 
Debes, J. H., Ge, J., \& Ftaclas, C. 2006, AJ, accepted, 131

Debes, J. H. \& Sigurdsson, S. 2002, ApJ, 572, 556

Debes, J. H., Sigurdsson, S., \& Woodgate, B. E. 2005a, ApJ, 633, 1168

-. 2005b, AJ, 130, 1221

Di Matteo, T., Carilli, C. L., \& Fabian, A. C. 2001, ApJ, 547, 731

Dobbie, P. D., Burleigh, M. R., Levan, A. J., Barstow, M. A., Napiwotzki, R., Holberg, J. B., Hubeny, I., \& Howell, S. B. 2005, MNRAS, 357, 1049

Dupuis, J., Fontaine, G., Pelletier, C., \& Wesemael, F. 1992, ApJS, 82, 505

-. 1993a, ApJS, 84, 73

Dupuis, J., Fontaine, G., \& Wesemael, F. 1993b, ApJS, 87, 345

Durney, B. R., De Young, D. S., \& Roxburgh, I. W. 1993, Sol. Phys., 145, 207

Farihi, J., Becklin, E. E., \& Zuckerman, B. 2005, ApJS, 161, 394

Farihi, J. \& Christopher, M. 2004, AJ, 128, 1868

Farihi, J., Hoard, D. W., \& Wachter, S. 2006, ApJ, 646, 480

Gianninas, A., Dufour, P., \& Bergeron, P. 2004, ApJ, 617, L57

Graham, J. R., Matthews, K., Neugebauer, G., \& Soifer, B. T. 1990, ApJ, 357, 216

Holberg, J. B., Barstow, M. A., \& Green, E. M. 1997, ApJ, 474, L127+

Houdebine, E. R., Foing, B. H., \& Rodono, M. 1990, A\&A, 238, 249

Jura, M. 2003, ApJ, 584, L91

Kawaler, S. D. 1988, ApJ, 333, 236

Koester, D., Provencal, J., \& Shipman, H. L. 1997, A\&A, 320, L57

Koester, D., Rollenhagen, K., Napiwotzki, R., Voss, B., Christlieb, N., Homeier, D., \& Reimers, D. 2005, A\&A, 432, 1025

Koester, D. \& Wilken, D. 2006, A\&A, 453, 1051

Krumholz, M. R., McKee, C. F., \& Klein, R. I. 2005, ApJ, 618, 757 
Lamers, H. J. G. L. M. \& Cassinelli, J. P. 1999, Introduction to Stellar Winds (Introduction to Stellar Winds, by Henny J. G. L. M. Lamers and Joseph P. Cassinelli, pp. 452. ISBN 0521593980. Cambridge, UK: Cambridge University Press, June 1999.)

Laughlin, G., Bodenheimer, P., \& Adams, F. C. 2004, ApJ, 612, L73

Liebert, J., Bergeron, P., \& Holberg, J. B. 2005, ApJS, 156, 47

Lim, J. \& White, S. M. 1996, ApJ, 462, L91+

López-Morales, M. \& Ribas, I. 2005, ApJ, 631, 1120

McCook, G. P. \& Sion, E. M. 1999, ApJS, 121, 1

Mestel, L. \& Spruit, H. C. 1987, MNRAS, 226, 57

Mohanty, S. \& Basri, G. 2003, ApJ, 583, 451

Mullan, D. J., Doyle, J. G., Redman, R. O., \& Mathioudakis, M. 1992, ApJ, 397, 225

Paquette, C., Pelletier, C., Fontaine, G., \& Michaud, G. 1986, ApJS, 61, 197

Perna, R., Narayan, R., Rybicki, G., Stella, L., \& Treves, A. 2003, ApJ, 594, 936

Plavchan, P., Jura, M., \& Lipscy, S. J. 2005, ApJ, 631, 1161

Probst, R. G. \& Oconnell, R. W. 1982, ApJ, 252, L69

Ritter, H. \& Kolb, U. 2003, A\&A, 404, 301

Saffer, R. A., Wade, R. A., Liebert, J., Green, R. F., Sion, E. M., Bechtold, J., Foss, D., \& Kidder, K. 1993, AJ, 105, 1945

Schreiber, M. R. \& Gänsicke, B. T. 2003, A\&A, 406, 305

Schultz, G., Zuckerman, B., \& Becklin, E. E. 1996, ApJ, 460, 402

Shimanskii, V. V. \& Borisov, N. V. 2002, Astronomy Reports, 46, 406

Sills, A., Pinsonneault, M. H., \& Terndrup, D. M. 2000, ApJ, 534, 335

Theuns, T., Boffin, H. M. J., \& Jorissen, A. 1996, MNRAS, 280, 1264

Theuns, T. \& Jorissen, A. 1993, MNRAS, 265, 946

Wargelin, B. J. \& Drake, J. J. 2002, ApJ, 578, 503 
Weber, E. J. \& Davis, L. J. 1967, ApJ, 148, 217

Wood, B. E., Linsky, J. L., Müller, H.-R., \& Zank, G. P. 2001, ApJ, 547, L49

Wood, B. E., Müller, H.-R., Zank, G. P., \& Linsky, J. L. 2002, ApJ, 574, 412

Zuckerman, B. \& Becklin, E. E. 1987, Nature, 330, 138

-. 1992, ApJ, 386, 260

Zuckerman, B., Koester, D., Reid, I. N., \& Hünsch, M. 2003, ApJ, 596, 477

Zuckerman, B. \& Reid, I. N. 1998, ApJ, 505, L143 
Table 1. Table of Close Binary Parameters

\begin{tabular}{ccccccc}
\hline \hline WD & $\begin{array}{c}\text { Period } \\
(\mathrm{d})\end{array}$ & $\begin{array}{c}\text { Orbital Separation } \\
(\mathrm{AU})\end{array}$ & $\begin{array}{c}\mathrm{M}_{W D} \\
\left(\mathrm{M}_{\odot}\right)\end{array}$ & $\begin{array}{c}\mathrm{M}_{R D} \\
\left(\mathrm{M}_{\odot}\right)\end{array}$ & $\begin{array}{c}\mathrm{R}_{R D} \\
\left(\mathrm{R}_{\odot}\right)\end{array}$ & Reference \\
\hline $0419-487$ & 0.3037 & $7.3 \times 10^{-3}$ & 0.47 & 0.095 & 0.189 & 1 \\
$1026+002$ & 0.597259 & 0.013 & 0.68 & 0.23 & 0.25 & 2 \\
$1213+528$ & 0.667579 & 0.015 & 0.63 & 0.36 & 0.32 & 3 \\
\hline
\end{tabular}

References. - (1) Bruch (1999), (2)Saffer et al. (1993), (3)Shimanskii \& Borisov (2002)

Table 2. Table of Wide Binary Parameters

\begin{tabular}{cccc}
\hline \hline WD & Ang. Separation & $\begin{array}{c}\text { Distance } \\
(\mathrm{pc})\end{array}$ & $\begin{array}{c}\text { Orbital Separation } \\
(\mathrm{AU})\end{array}$ \\
\hline $0354+463$ & $<0.2^{\prime \prime}$ & $30^{\mathrm{a}}$ & $1.5 \pm 0.5$ \\
$1049+103$ & $0.26^{\prime \prime}$ & $107^{\mathrm{b}}$ & 28 \\
$1210+464$ & $1.04^{\prime \prime}$ & $153^{\mathrm{b}}$ & 159 \\
\hline
\end{tabular}

${ }^{a}$ McCook \& Sion (1999)

${ }^{\mathrm{b}}$ Liebert et al. (2005) 
Table 3. WD Parameters and Derived Mass Loss Rates

\begin{tabular}{ccccccc}
\hline \hline WD & $\begin{array}{c}\mathrm{T}_{\text {eff }} \\
(\mathrm{K})\end{array}$ & {$[\mathrm{Ca} / \mathrm{H}]$} & $\log q$ & $\begin{array}{c}\log \Theta \\
(\mathrm{yr})\end{array}$ & $\begin{array}{c}\dot{M} \\
\left(\mathrm{M}_{\odot} / \mathrm{yr}\right)\end{array}$ & $\begin{array}{c}\dot{M}_{R D} \\
\left(\mathrm{M}_{\odot} / \mathrm{yr}\right)\end{array}$ \\
\hline $0354+463$ & 7765 & -8.335 & -8.8 & 3.37 & $6 \times 10^{-16}$ & $6 \times 10^{-10}$ \\
$0419-487$ & 6296 & -9.284 & -7 & 4.5 & $4 \times 10^{-16}$ & $6 \times 10^{-15}$ \\
$1026+002$ & 14300 & -8.56 & -16.6 & -2 & $2 \times 10^{-18}$ & $1 \times 10^{-16}$ \\
$1049+103$ & 19800 & -7.182 & $<-18$ & $<-2.2$ & $3 \times 10^{-18}$ & $7 \times 10^{-10}$ \\
$1210+464$ & 27000 & -7 & $<-18$ & $<-2.2$ & $4 \times 10^{-18}$ & $5 \times 10^{-8}$ \\
$1213+528$ & 13000 & -8.1 & -16 & -0.6 & $9 \times 10^{-19}$ & $1 \times 10^{-16}$ \\
\hline \hline Proxima Centauri & & & & & $4 \times 10^{-15}, 6 \times 10^{-14}$ \\
\hline
\end{tabular}



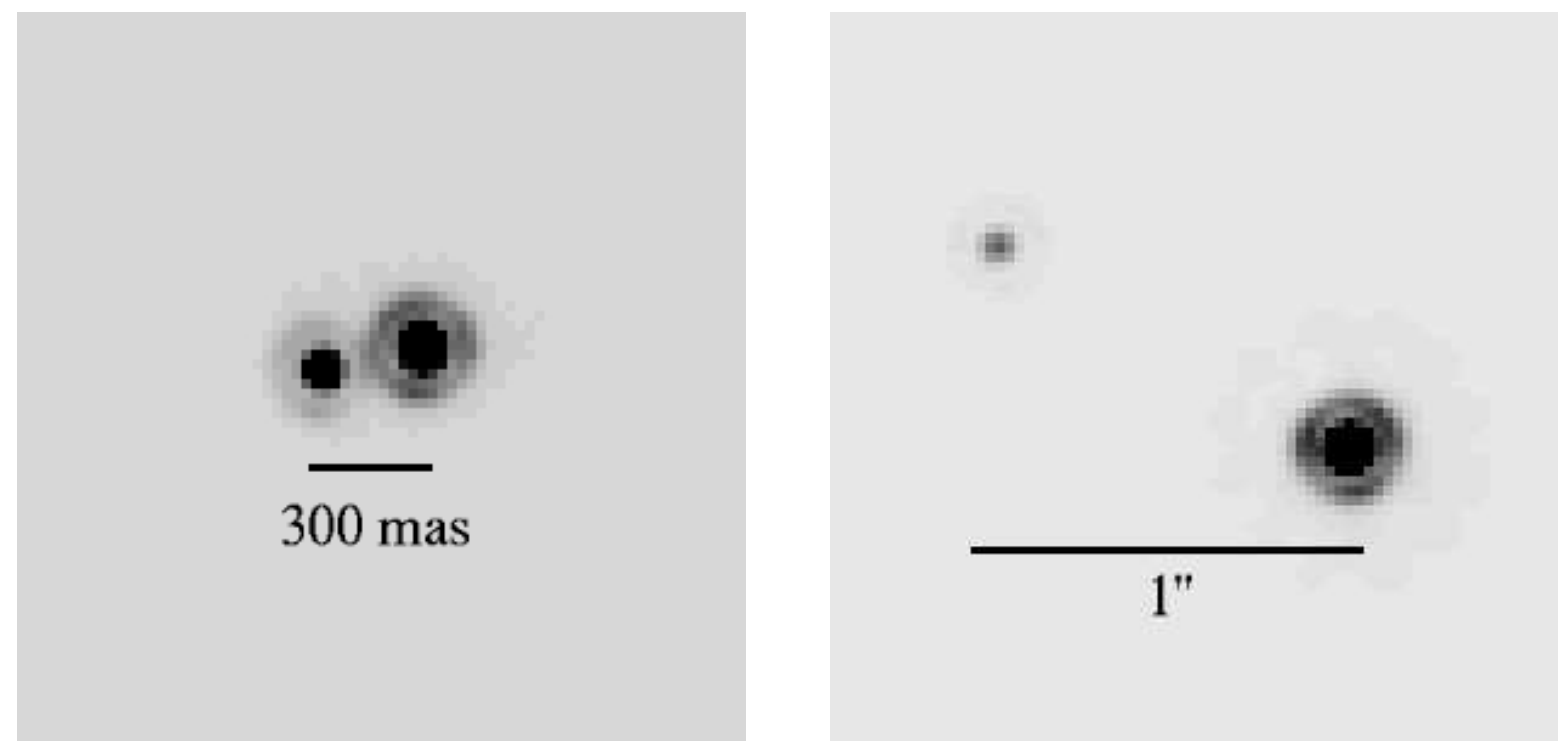

Fig. 1.- (left)ACS HRC image of WD 1049+103 with its resolved M dwarf companion. (right) ACS HRC image of WD 1210+464 with its resolved M dwarf companion.
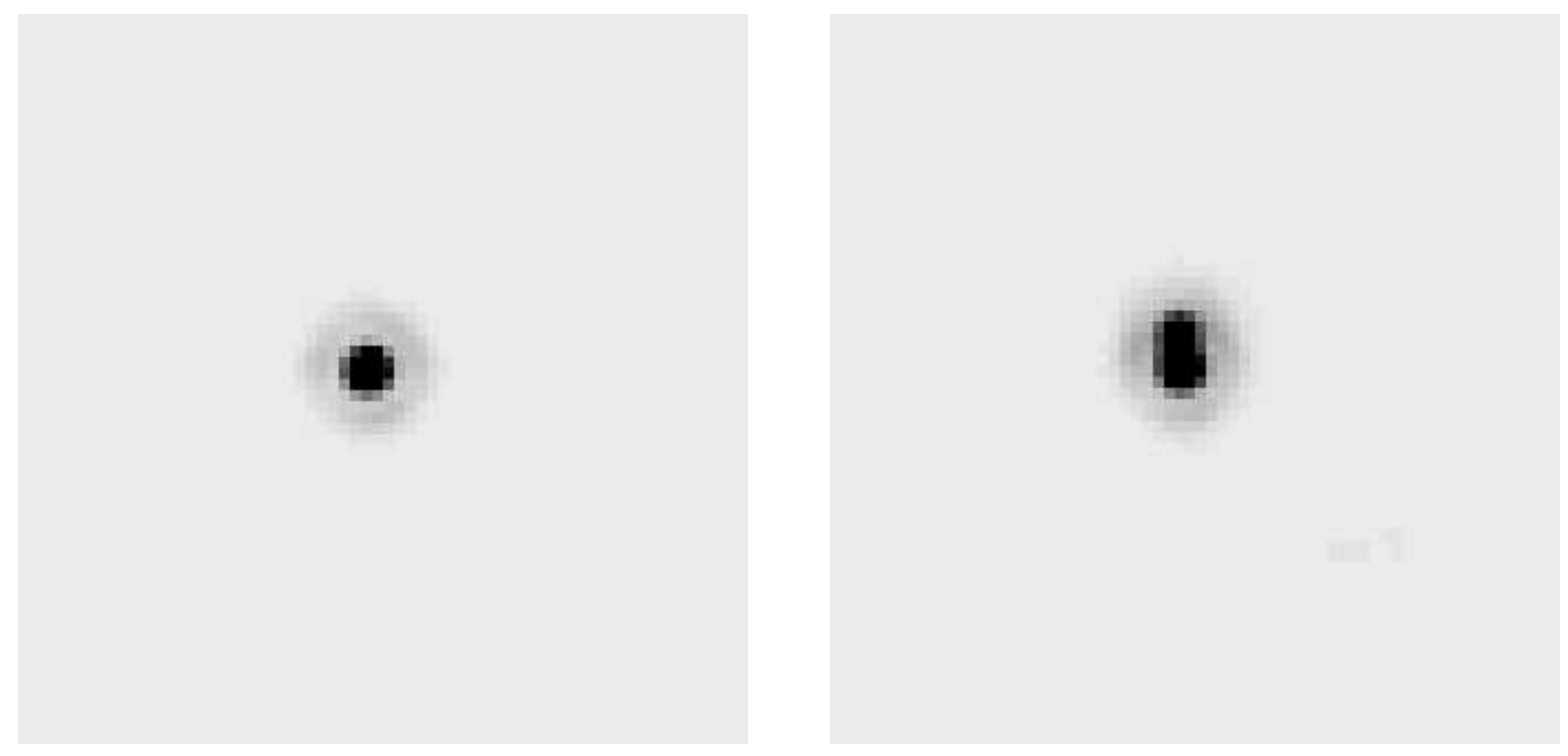

Fig. 2.- (left) ACS HRC image of WD 0354+463 without any artificial implants. (right) Same image, but with an artificial companion with $\mathrm{m}_{F 814 W}=15.4$ at a separation of 70 mas. 


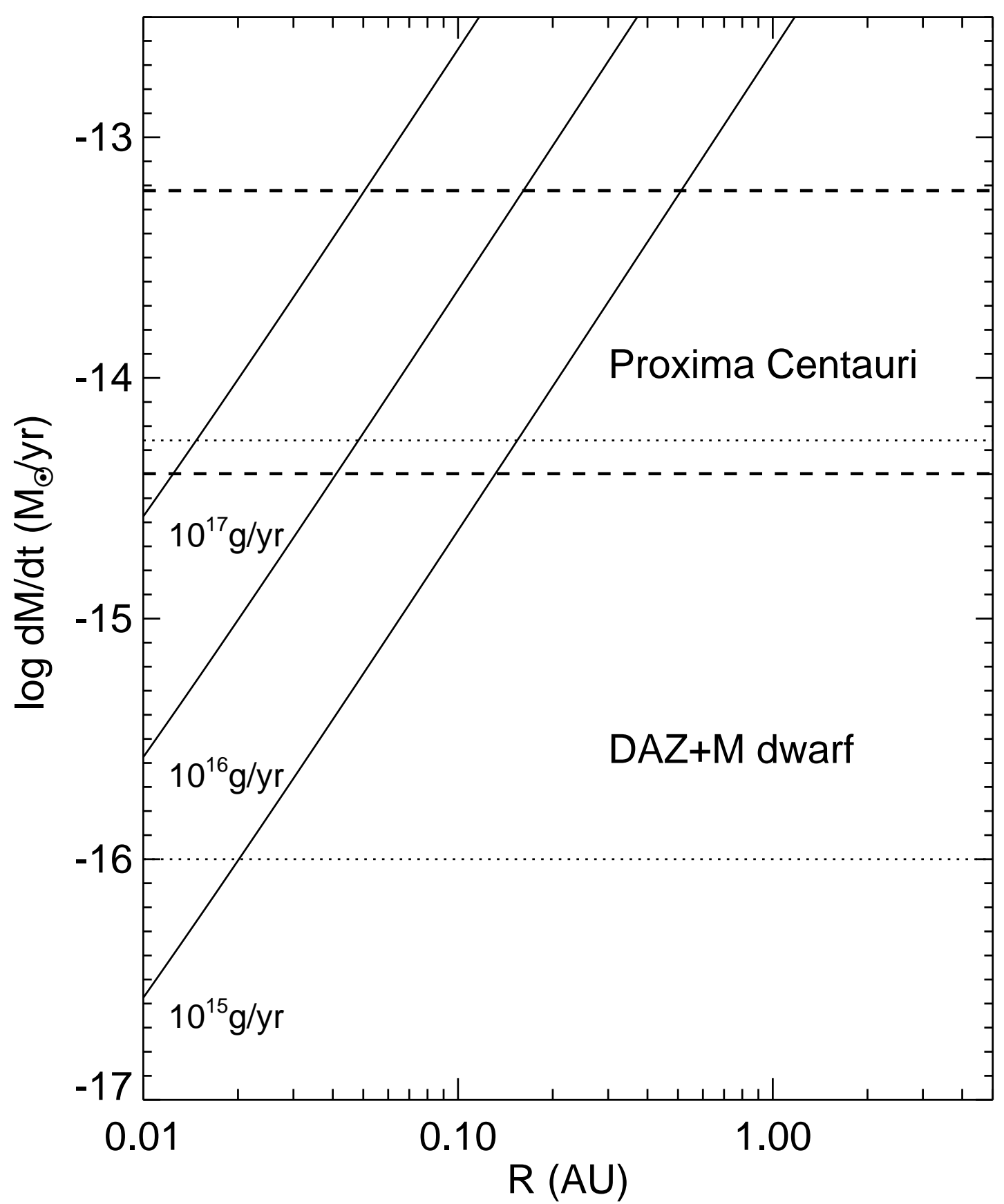

Fig. 3.- Calculation of stellar mass loss rates for given observed white dwarf accretion rates as a function of distance. Each curve corresponds to a different level of accretion one would observe on a white dwarf. The horizontal dashed lines encompass the range of mass loss rate values observed as upper limits for Proxima Centauri and the dash-dotted lines encompass the rates calculated for the DAZ $+\mathrm{M}$ dwarf sample 